\title{
Evidenzbasiertes Tarifmonitoring im praxisambulanten Bereich
}

\author{
Kerstin Schutz, Sabine Zehnder
}

Expertinnen Abteilung Ambulante Versorgung und Tarife

\author{
Kostenentwicklungen im Gesundheitswesen werden von verschiedenen Akteuren \\ diskutiert, jedoch unterschiedlich interpretiert. Die FMH kann mit der ärzteeige- \\ nen Datensammlung Entwicklungen im praxisambulanten Sektor analysieren und \\ in einen medizinischen Kontext setzen.
}

Die Entwicklung der Gesundheitskosten ist seit Jahren Teil der politischen Agenda. Als Vertreterin der Ärztinnen und Ärzte in der Schweiz ist es eine Aufgabe der FMH, Entwicklungen zu verfolgen und einzuordnen. Die Entwicklung von praxisambulanten Kosten kann unterschiedliche Ursachen haben und ist nicht zwangsläufig eine arztinduzierte Mengenausweitung.

\section{In den letzten drei Jahren haben ca. 10000 Arztpraxen aktiv Daten geliefert.}

Das Herzstück des FMH-Tarifmonitorings bildet die ärzteeigene Datensammlung. In den letzten drei Jahren haben ca. 10000 Arztpraxen aktiv Daten geliefert und durch die Beiträge an die kantonalen Trustcenter die Datensammlung finanziert. Nachdem die Société Vaudoise de Médecine (SVM) im Kanton Waadt und Teile der Innerschweiz eigene Lösungen verfolgen, rechnen wir im praxisambulanten Bereich mit einer schweizweiten Abdeckung von rund 65\%. Im Datenpool der Schweizer Ärzteschaft sind aktuell 275 Mio. Rechnungskopien mit knapp 2,8 Milliarden Leistungsdatensätzen (Stand: Mitte Juni 2019) vorhanden. Damit sind die Daten grundsätzlich in jeder auf einer Rechnung verfügbaren Dimension auswertbar. Die Daten werden durch NewIndex/TrustX aufbereitet und der FMH in anonymisierter Form für Analysen zur Verfügung gestellt. Der Datenschutz ist immer gewährleistet, und der NAKO-Kodex ${ }^{1}$ wird strikte eingehalten.

Die FMH verfolgt in ihren Analysen die Entwicklungen "pro Einheit» auf relativer Ebene, beispielsweise pro Patient, pro Praxis oder pro Sitzung. So können exogene Effekte bei der Analyse möglicher Ursachen vernachlässigt werden. Damit sind die analysierten
Daten aussagekräftiger als beispielsweise die Wachstumsrate der Gesamtkosten. Der Blick auf die Gesamtkosten oder auf die Basis des Gesamtvolumens an Taxpunkten ist ohne Frage relevant, jedoch nur ein Aspekt einer ganzheitlichen Betrachtung. Zudem lässt diese Betrachtungsweise viele exogene Faktoren ausser Acht, wie beispielsweise das Bevölkerungswachstum oder politische Vorgaben wie "Ambulant vor Stationär».

Unter anderem werden folgende Kennzahlen analysiert und kontextualisiert:

- Volumen pro Sitzung (TARMED)

Darunter sind die Kosten (Anzahl Taxpunkte) einer durchschnittlichen Sitzung zu verstehen. Zur besseren Übersicht werden die Tarifpositionen in verschiedene Blöcke zusammengefasst, z.B. in typische Leistungen einer Grundkonsultation. Diese Art der Analyse erlaubt einen differenzierten Blick auf die Volumenveränderungen von Tarifpositionen und setzt diese in Relation zu anderen Entwicklungen. Damit lassen sich etwa als «beängstigend» wahrgenommene Effekte, die aber nur einen marginalen Anteil am Gesamtvolumen haben, relativieren oder umgekehrt der Einfluss scheinbar geringer Veränderungen auf das Gesamtvolumen erkennen.

- Volumen pro Patient (TARMED)

Das Volumen pro Patient gibt die Kosten (Anzahl Taxpunkte) pro Patient an, welche durch einen Arzt oder eine Ärztin innerhalb einer Periode im TARMED abgerechnet worden sind.

- Volumen pro Praxis (TARMED)

Das Volumen pro Praxis gibt die durchschnittlichen Kosten (Anzahl Taxpunkte) einer Arztpraxis innerhalb einer Periode an. Als Arztpraxis gilt eine ZSRNummer. 


\section{- Sitzungen pro Patient}

Die Anzahl Sitzungen pro Patient gibt an, wie oft ein Patient innerhalb einer Periode bei einem Arzt/einer Ärztin in Behandlung war. Als Sitzung gilt ein Patientenkontakt pro Tag.

- Sitzungen pro Praxis

Die Anzahl Sitzungen pro Praxis zählt die Anzahl Sitzungen über alle Patienten einer Arztpraxis in einer Periode. Als Arztpraxis gilt eine ZSR-Nummer.

- Patienten pro Praxis

Die Anzahl Patienten pro Praxis gibt an, wie viel Patienten (Erstkontakte) innerhalb einer Periode bei einem Arzt oder einer Ärztin in Behandlung waren. Als Arztpraxis gilt eine ZSR-Nummer.

\section{Kontextualisierung}

Mit diesen Kennzahlen können verschiedene Effekte analysiert werden:

In der untenstehenden Grafik ist beispielsweise ein Anstieg aller Sitzungen seit 2016 zu verzeichnen. Diese Entwicklung wird allerdings relativiert durch die Anzahl Patienten (Erstkontakte), die pro Praxis ebenfalls angestiegen ist.

\section{Über die letzten sechs Jahre wurde eine Sitzungszunahme pro Patient von durch- schnittlich $0,64 \%$ pro Jahr beobachtet.}

Die Anzahl Sitzungen pro Patient nimmt 2019 um $0,62 \%$ marginal zu im Vergleich zum Vorjahr. Über die letzten sechs Jahre wurde eine Sitzungszunahme pro Patient von durchschnittlich 0,64\% pro Jahr beobachtet. Damit liegt der Anstieg im Durchschnitt der letzten Jahre. Ärztinnen und Ärzte bieten Patienten also nicht zwangsläufig häufiger auf als vor dem Tarifeingriff 2018.

Das TARMED-Volumen pro Sitzung sinkt 2019 weiter um weitere $-0,26 \%$ (2018: $-3,69 \%)$.
Das TARMED-Volumen pro Patient zeigt 2019 einen unterdurchschnittlichen Anstieg von 0,38\% im Vergleich zum Vorjahr. Der Durchschnitt über die letzten sechs Jahre liegt bei einem Anstieg von 1,71\% pro Patient.

Um Analysen miteinander vergleichen zu können, ist die Kenntnis der Definitionen und der Basis des Datenmaterials vonnöten.

\section{Vergleichbarkeit: Behandlungsdatum und Verarbeitungsdatum}

Das Monitoring der Krankenversicherungs-Kostenentwicklung (MOKKE) des Bundesamtes für Gesundheit BAG wertet beispielsweise ebenfalls Kosten pro Einheit aus, in diesem Fall die Gesamtkosten pro versicherte Person, in verschiedenen Sektoren und über alle Leistungserbringer. Dieses Monitoring basiert auf Krankenversicherungsdaten, genauer gesagt dem Datum, an welchem die entsprechende Leistung innerhalb der Krankenversicherung verarbeitet worden ist (Verarbeitungsdatum).

Die Analysen der FMH beruhen im Gegensatz dazu auf dem Behandlungsdatum, d.h. dem Tag, an dem die Leistung erbracht worden ist. So können auch saisonale Effekte oder aussergewöhnliche Ereignisse genauer analysiert werden. Beide Analysemethoden ergänzen sich damit. Die Betrachtung der FMH ist die betriebswirtschaftliche Sicht der Arztpraxis, während MOKKE eher eine volkswirtschaftliche Betrachtung vornimmt.

\section{Vergleichbarkeit: Erkrankte und Versicherte}

Ein weiteres Beispiel für eine Vorsicht bei Vergleichen beispielsweise mit MOKKE-Daten ist die Einheit «Versicherte» (MOKKE) und «Erkrankte/Patienten» (FMHMonitoring). Die Kosten pro Versicherten ist die

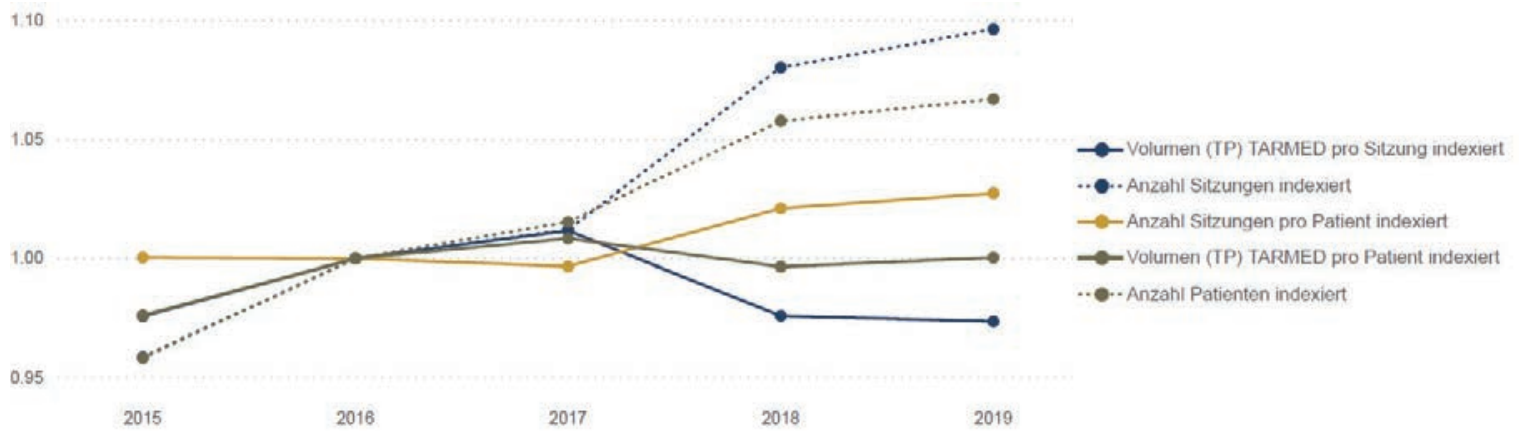

Abbildung 1: Entwicklungen tarifrelevanter Kennzahlen im praxisambulanten Bereich (Indexjahr 2016). 
FMH/Abteilung Ambulante Versorgung und Tarife Baslerstrasse 47 CH-4600 Olten

Tel. 0313591230 Fax 0313591238 tarife.ambulant[at]fmh.ch
Summe aller ambulanten ärztlichen Behandlungen (TARMED, verhandelte Pauschalen etc.) von mehreren Leistungserbringern.

Das Taxpunktvolumen pro Patient (Erkrankten) des FMH-Monitorings weist hingegen das Taxpunktvolumen pro Patient bei einem Leistungserbringer aus. Wenn ein Patient beispielsweise in einer Periode seinen Hausarzt und einen Spezialisten aufsucht, werden die Kosten für den Hausarzt und den Spezialisten im FMH-Monitoring für diesen Patienten separat pro Arzt (ZSR-Nummer) ausgewiesen, und damit wird die Person als zwei Erkrankte für diese Periode betrachtet.

\section{Differenzierte Betrachtung und weitere Messgrössen}

Die ärzteeigenen Daten erlauben einen differenzierten Blick auf Entwicklungen von Abrechnungsdaten im praxisambulanten Bereich.

Neben den vorgestellten Kennzahlen werden viele weitere Analysen durchgeführt: Unter anderem, wie sich die Zusammensetzung einer durchschnittlichen Sitzung verändert oder auch welche Leistungen gemeinsam in einer Sitzung erbracht werden (Co-Positionen).
Des Weiteren können differenzierte Analysen für verschiedene Patientengruppen vorgenommen werden (zum Beispiel nach Alter und Geschlecht). Neben den Auswertungen zum Arzttarif TARMED werden Analysen zum Abrechnungsvolumen der Analysenliste (Pra-

Die umfassenden Analysen sind nur dank der Arztpraxen möglich, die ihre Abrechnungsdaten an die kantonalen Trustcenter liefern.

xislabor) und von Medikamenten durchgeführt.

Die FMH bezieht selbstverständlich auch andere Publikationen in ihre Analysen ein.

Die umfassenden Analysen sind nur dank jener Arztpraxen möglich, welche ihre Abrechnungsdaten an die kantonalen Trustcenter liefern. Die Zahl der Datenlieferanten nimmt dabei stetig zu. Wir bedanken uns bei jedem Arzt und jeder Ärztin, die mit der Datenlieferung die Datensammlung speisen, finanzieren und somit dazu beitragen, dass die FMH in der Lage ist, solche Analysen durchzuführen.

Ein weiterer Dank geht an die Ärztekasse, die uns mit ihrem grossen Know-how bei der Ausarbeitung und Aufbereitung dieses Monitorings aktiv unterstützt und beraten hat.

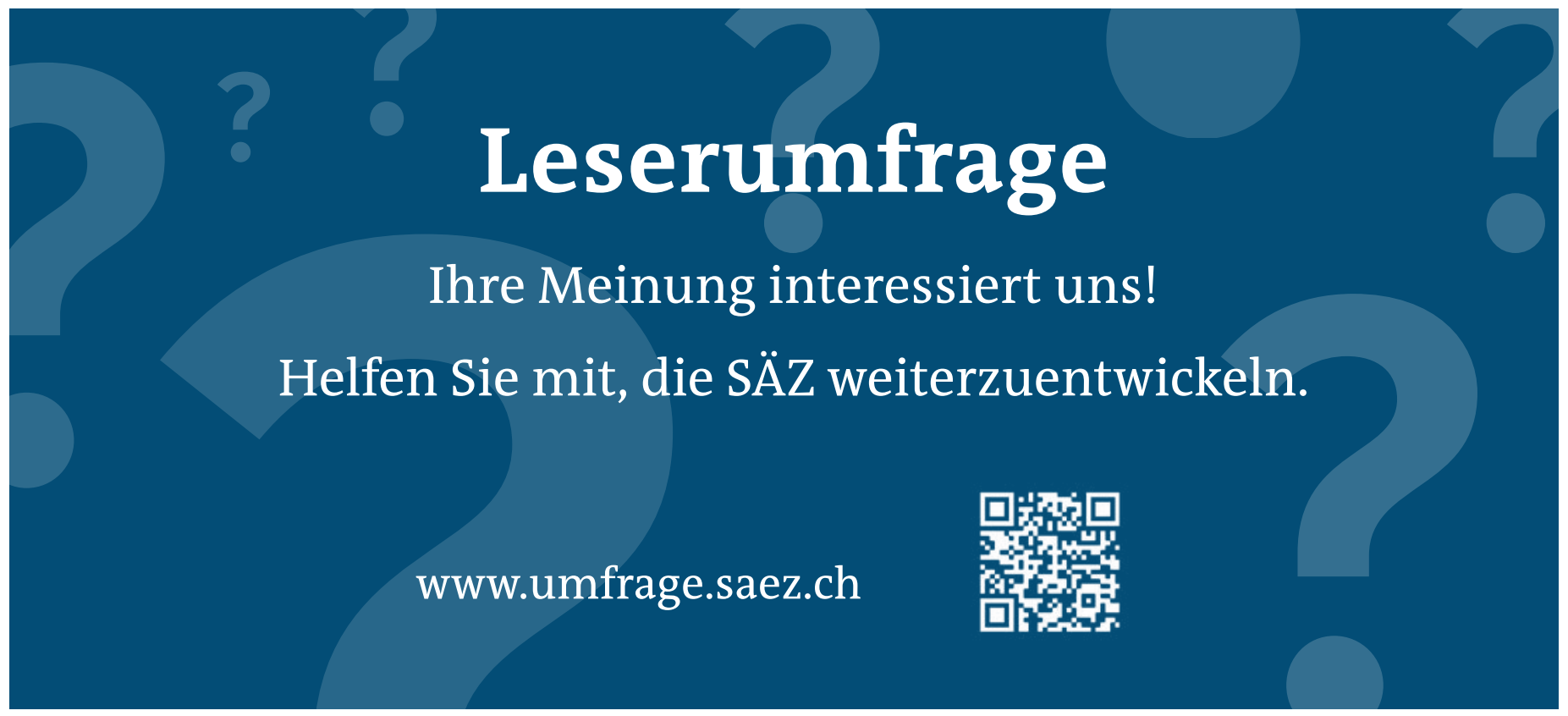

